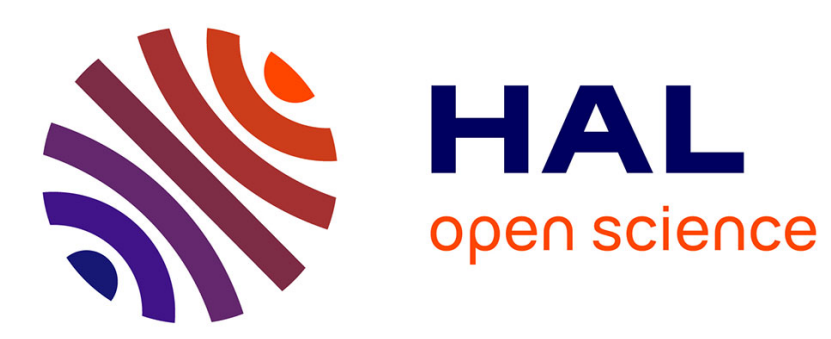

\title{
Fast and Faithful Performance Prediction of MPI Applications: the HPL Case Study
}

\author{
Tom Cornebize, Arnaud Legrand, Franz C Heinrich
}

\section{To cite this version:}

Tom Cornebize, Arnaud Legrand, Franz C Heinrich. Fast and Faithful Performance Prediction of MPI Applications: the HPL Case Study. 2019. hal-02096571v3

\section{HAL Id: hal-02096571 \\ https://hal.inria.fr/hal-02096571v3}

Preprint submitted on 27 May 2019 (v3), last revised 26 Sep 2019 (v4)

HAL is a multi-disciplinary open access archive for the deposit and dissemination of scientific research documents, whether they are published or not. The documents may come from teaching and research institutions in France or abroad, or from public or private research centers.
L'archive ouverte pluridisciplinaire HAL, est destinée au dépôt et à la diffusion de documents scientifiques de niveau recherche, publiés ou non, émanant des établissements d'enseignement et de recherche français ou étrangers, des laboratoires publics ou privés. 


\title{
Fast and Faithful Performance Prediction of MPI Applications: the HPL Case Study
}

\author{
Tom Cornebize, Arnaud Legrand, Franz C. Heinrich \\ Univ. Grenoble Alpes, CNRS, Inria, Grenoble INP, LIG, 38000 Grenoble, France \\ firstname.lastname@inria.fr
}

Table I: For large matrices, simulation is orders of magnitude less expensive than real executions

\begin{tabular}{|c|c|c|c|}
\hline & & Reality & Simulation \\
\hline \multirow{4}{*}{ 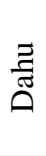 } & \#nodes / \#processes & $32 / 1024$ & $1 / 1$ \\
\hline & Memory & $2 \mathrm{~TB}$ & $9 \mathrm{~GB}$ \\
\hline & Duration (hours) & 1 & 5 \\
\hline & Resources (node hours) & 32 & 1 \\
\hline \multirow{4}{*}{ 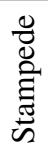 } & \#nodes / \#processes & 6006 / 6006 & $1 / 1$ \\
\hline & Memory & $120 \mathrm{~TB}$ & $19 \mathrm{~GB}$ \\
\hline & Duration (hours) & 2 & 62 \\
\hline & Resources (node hours) & 12,000 & 62 \\
\hline
\end{tabular}

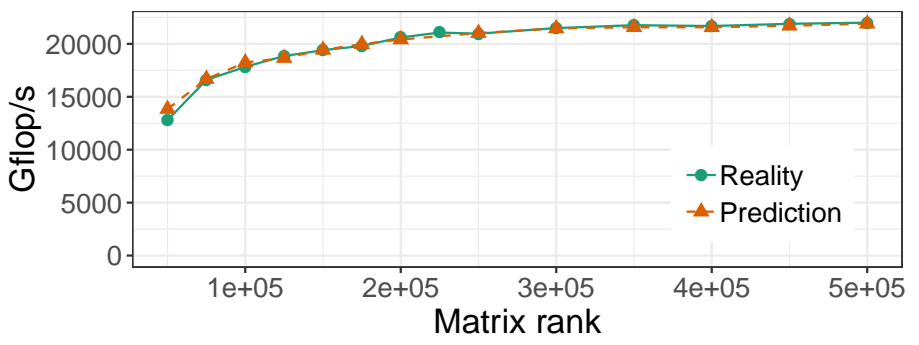

Figure 1: HPL performance on Dahu can be predicted within a few percents of reality using a stochastic heterogeneous model for dgemm and a simple model for all other kernels and the network

\begin{abstract}
Finely tuning MPI applications (number of processes, granularity, collective operation algorithms, geometry and process placement) is critical to obtain good performance on supercomputers. With a rising cost of modern supercomputers, running parallel applications at scale solely to optimize their performance is extremely expensive. Having inexpensive but faithful predictions of expected performance could be a great help for researchers and system administrators. The methodology we propose captures the complexity of adaptive applications by emulating the MPI code while skipping insignificant parts. We demonstrate its capability with High Performance Linpack (HPL), the benchmark used to rank supercomputers in the TOP500 and which requires a careful tuning. We explain how we both (1) extended the SimGrid's SMPI simulator and slightly modified the open-source version of HPL to allow a fast emulation on a single commodity server at the scale of a supercomputer and (2) how to model the different components (network, BLAS, ...) of the platform. We show that a careful modeling of both spatial and temporal node variability allows us to obtain predictions within a few percents of real experiments (see Figure 1).
\end{abstract}

\section{INTRODUCTION}

Today, machines with 100,000 cores and more are common and several machines beyond the 1,000,000 cores mark are already in production. This high density of computation units requires a diligent optimization of application parameters, such as problem size, process organization or choice of algorithm, as these have a huge impact on load distribution and network utilization. Scientific application developers and users often spend a considerable amount of time and effort running their application at different scales solely to tweak parameters and therefore optimize their performance. Whenever actual performance does not match expectations, it can be very difficult to understand whether the mismatch originates from application misunderstanding or machine misconfiguration. This expenditure of time, combined with the power consumption that often reaches several MW, makes it financially expensive to test-run applications. Similar difficulties are encountered when (co-)designing supercomputers for specific applications. A large part of this tuning work could be simplified if a generic and faithful performance prediction tool was available. This article presents a decisive step in this direction.

In this article, we explain how to predict the performance of High-Performance Linpack (HPL) on a supercomputer with the SimGrid/SMPI [1], [2] simulator. HPL is used to rank twice a year the world's largest and fastest machines in the TOP500 [3] and requires both a careful tuning and a sizable input to get near to the peak performance. We detail how we obtained faithful models for several key functions (e.g., dgemm and dtrsm) and managed to reduce the memory consumption from more than a hundred terabytes to several gigabytes. This allowed us to emulate HPL on a single commodity server within 1-3 days scenarios similar to the run that was carried out on the Stampede cluster (hosted at TACC) in 2013 for the TOP500 (see Table I). More importantly, we show that despite the genericity of our approach (we only modify a few dozens of lines of HPL), our simulation allows to predict the performance of HPL on a recent cluster (running a thousand MPI ranks) within a few percent of reality (see Figure 1).

This article is organized as follows: Section II presents the main characteristics of the HPL application and provides information on how the runs are conducted on modern supercomputers. Section III discusses related work and explains why emulation (or online simulation) is the only sensible approach when studying an application as complex as HPL. In Section IV, we briefly present the simulator we used for this work, SimGrid/SMPI, followed by an extensive discussion in Section V about the optimizations on all levels (i.e., simulator, application, operating system) that are necessary to make a 


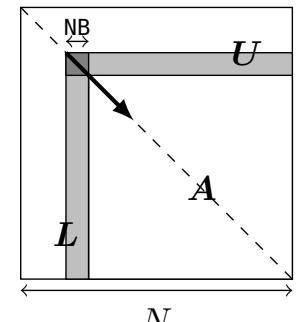

$$
\begin{aligned}
& \text { allocate and initialize } A \\
& \text { for } k=N \text { to } 0 \text { step } N B \text { do } \\
& \begin{array}{l}
\text { allocate the panel } \\
\text { factor the panel } \\
\text { broadcast the panel } \\
\text { update the sub-matrix; }
\end{array}
\end{aligned}
$$

Figure 2: Overview of High Performance Linpack

large-scale run tractable. The scalability of our approach is evaluated in Section V-C. In Section VI, we explain how the different resources of the supercomputer (network, compute kernels) can be modeled to predict the performance of HPL faithfully. We particularly discuss the usefulness and relevance of different degrees of modeling complexity. In Section VII, we compare simulation results with real experiments and illustrate the importance of modeling both spatial and temporal variability. Section VIII concludes this article by discussing perspectives and future work.

\section{CONTEXT}

\section{A. High-Performance Linpack}

In this work, we use the freely-available referenceimplementation of HPL [4], which relies on MPI. HPL implements a matrix factorization based on a right-looking variant of the LU factorization with row partial pivoting and allows multiple look-ahead depths. The principle of the factorization is depicted in Figure 2. It consists of a series of panel factorizations followed by an update of the trailing sub-matrix. HPL uses a two-dimensional block-cyclic data distribution of $A$ and implements several custom MPI collective communication algorithms to efficiently overlap communications with computations. The main parameters of HPL are:

- $N$ is the order of the square matrix $A$.

- NB is the "blocking factor", i.e., the granularity at which HPL operates when panels are distributed or worked on.

- $P$ and $Q$ denote the number of process rows and the number of process columns, respectively.

- RFACT determines the panel factorization algorithm. Possible values are Crout, left- or right-looking.

- SWAP specifies the swapping algorithm used while pivoting. Two algorithms are available: one based on binary exchange (along a virtual tree topology) and the other one based on a spread-and-roll (with a higher number of parallel communications). HPL also provides a panel-size threshold triggering a switch from one variant to the other.

- BCAST sets the algorithm used to broadcast a panel of columns over the process columns. Legacy versions of the MPI standard only supported non-blocking point-topoint communications, which is why HPL ships with in total 6 self-implemented variants to overlap the time spent waiting for an incoming panel with updates to the trailing matrix: ring, ring-modified, 2-ring, 2-ring-modified, long, and long-modified. The modified versions guarantee that the process right after the root (i.e., the process that will become the root in the next iteration) receives data first and does not further participate in the broadcast. This process can thereby start working on the panel as soon as possible. The ring and 2-ring versions each broadcast along the corresponding virtual topologies while the long version is a spread and roll algorithm where messages are chopped into $Q$ pieces. This generally leads to better bandwidth exploitation. The ring and 2-ring variants rely on MPI_Iprobe, meaning they return control if no message has been fully received yet, hence facilitating partial overlap of communication with computations. In HPL 2.1 and 2.2, this capability has been deactivated for the long and longmodified algorithms. A comment in the source code states that some machines apparently get stuck when there are too many ongoing messages.

- DEPTH controls how many iterations of the outer loop can overlap with each other.

The sequential complexity of this factorization is flop $(N)=\frac{2}{3} N^{3}+2 N^{2}+\mathcal{O}(N)$ where $N$ is the order of the matrix to factorize. The time complexity can be approximated by

$$
T(N) \approx \frac{\left(\frac{2}{3} N^{3}+2 N^{2}\right)}{P \cdot Q \cdot w}+\Theta\left((P+Q) \cdot N^{2}\right),
$$

where $w$ is the flop rate of a single node and the second term corresponds to the communication overhead which is influenced by the network capacity and the previously listed parameters (RFACT, SWAP, BCAST, DEPTH, ...) and is very difficult to predict.

\section{B. Typical Runs on a Supercomputer}

Although the TOP500 reports precise information about the core count, the peak performance and the effective performance, it provides almost no information on how (software versions, HPL parameters, etc.) this performance was achieved. Some colleagues agreed to provide us with the HPL configuration they used and the output they submitted for ranking (see Table II). In June 2013, the Stampede supercomputer at TACC was ranked 6th in the TOP500 by

\begin{tabular}{|c|c|c|c|}
\hline & Stampede@TACC & Theta@ANL & Dahu@G5K \\
\hline Rpeak & 8520.1 TFlop s ${ }^{-1}$ & 9627.2 TFlop s $^{-1}$ & 62.26 TFlop s $^{-1}$ \\
\hline$N$ & $3,875,000$ & $8,360,352$ & 500,000 \\
\hline NB & 1024 & 336 & 128 \\
\hline$P \times Q$ & $77 \times 78$ & $32 \times 101$ & $32 \times 32$ \\
\hline RFACT & Crout & Left & Right \\
\hline SWAP & Binary-exch. & Binary-exch. & Binary-exch. \\
\hline BCAST & Long modified & 2 Ring modified & 2 Ring \\
\hline DEPTH & 0 & 0 & 1 \\
\hline Rmax & 5168.1 TFlop s ${ }^{-1}$ & 5884.6 TFlop s $^{-1}$ & 24.55 TFlop s $^{-1}$ \\
\hline Duration & 2 hours & 28 hours & 1 hour \\
\hline Memory & $120 \mathrm{~TB}$ & $559 \mathrm{~TB}$ & $2 \mathrm{~TB}$ \\
\hline MPI ranks & $1 /$ node & $1 /$ node & $1 /$ core \\
\hline
\end{tabular}
achieving 5168.1 TFlop s ${ }^{-1}$. In November 2017, the Theta supercomputer at ANL was ranked 18th with a performance of 5884.6 TFlop s $^{-1}$ but required a 28 -hour run on the whole machine. Finally, we ran HPL ourselves on a Grid' 5000 cluster named Dahu whose software stack could be fully controlled.

Table II: Typical runs of HPL 
The performance typically achieved by supercomputers (Rmax) needs to be compared to the much larger peak performance (Rpeak). This difference can be attributed to the node usage, to the MPI library, to the network topology that may be unable to deal with the intense communication workload, to load imbalance among nodes (e.g., due to a defect, system noise, ... ), to the algorithmic structure of HPL, etc. All these factors make it difficult to know precisely what performance to expect without running the application at scale. It is clear that due to the level of complexity of both HPL and the underlying hardware, simple performance models (analytic expressions based on $N, P, Q$ and estimations of platform characteristics as presented in Section II-A) may be able to provide trends but can by no means accurately predict the performance for each configuration (e.g., consider the exact effect of HPL's six different broadcast algorithms on network contention). Additionally, these expressions do not allow engineers to improve the performance through actively identifying performance bottlenecks. For complex optimizations such as partially non-blocking collective communication algorithms intertwined with computations, a very faithful modeling of both the application and the platform is required. Our goal in this article is to simulate systems at the scale of Stampede. Given the scale of this scenario (3,785 steps on 6,006 nodes in two hours), detailed simulations quickly become intractable without significant effort.

\section{RELATED WORK}

A first approach for estimating the performance of applications like HPL is statistical modeling of the application as a whole [5]. By running the application several times with small and medium problem sizes (of a few iterations of large problem sizes) and using simple linear regressions, it is possible to predict its makespan for larger sizes with an error of only a few percents and a relatively low cost. Unfortunately, the predictions are limited to the same application configuration and studying the influence of the number of rows and columns of the virtual grid or of the broadcast algorithms requires a new model and new (costly) runs using the whole target machine. Furthermore, this approach does not allow to study what-if scenarios (e.g., to evaluate what would happen if the network bandwidth was increased or if node heterogeneity was decreased) that are particularly useful when investigating potential performance improvements.

Simulation provides the details and flexibility missing to such black-box modeling approach. Performance prediction of MPI applications through simulation has been widely studied over the last decades but two approaches can be distinguished in the literature: offline and online simulation.

With the most common approach, offline simulation, a trace of the application is first obtained on a real platform. This trace comprises sequences of MPI operations and CPU bursts and is given as an input to a simulator that implements performance models for the CPUs and the network to derive predictions. Researchers interested in finding out how their application reacts to changes to the underlying platform can replay the trace on commodity hardware at will with different platform models. Most HPC simulators available today, notably BigSim [6], Dimemas [7] and CODES [8], rely on this approach. The main limitation of this approach comes from the trace acquisition requirement. Not only is a large machine required but the compressed trace of a few iterations (out of several thousands) of HPL typically reaches a few hundred MB, making this approach quickly impractical [9]. Worse, tracing an application provides only information about its behavior at the time of the run: slight modifications (e.g., to communication patterns) may make the trace inaccurate. The behavior of simple applications (e.g., stencil) can be extrapolated from small-scale traces [10], [11] but this fails if the execution is non-deterministic, e.g., whenever the application relies on non-blocking communication patterns, which is unfortunately the case for HPL.

The second approach discussed in the literature is online simulation. Here, the application is executed (emulated) on top of a simulator that is responsible to determine when each process is run. This approach allows researchers to study directly the behavior of MPI applications but only a few recent simulators such as SST Macro [12], SimGrid/SMPI [1] and the closed-source xSim [13] support it. To the best of our knowledge, only SST Macro and SimGrid/SMPI are mature enough to faithfully emulate HPL. In this work, we decided to rely on SimGrid as its performance models and its emulation capabilities seemed quite solid but the developments we propose would a priori also be possible with SST. Note that the HPL emulation we describe in Section V should not be confused with the application skeletonization [14] commonly used with SST. Skeletons are code extractions of the most important parts of a complex application whereas we only modify a few dozens of lines of HPL before emulating it with SMPI. Finally, it is important to understand that the approach we propose is intended to help studies at the level of the whole machine and application, not the influence of microarchitectural details as intended by MUSA [15].

\section{SIMGRID/SMPI IN A NUTSHELL}

SimGrid [1] is a flexible and open-source simulation framework that was originally designed in 2000 to study scheduling heuristics tailored to heterogeneous grid computing environments but has later been extended to study cloud and HPC infrastructures. The main development goal for SimGrid has been to provide validated performance models particularly for scenarios making heavy use of the network. Such a validation usually consists of comparing simulation predictions with results from real experiments to confirm or debunk network and application models.

SMPI, a simulator based on SimGrid, has been developed and used to simulate unmodified MPI applications written in $\mathrm{C} / \mathrm{C}++$ or FORTRAN [2]. The complex network optimizations done in real MPI implementations need to be considered when predicting the performance of MPI applications. For instance, the "eager" and "rendez-vous" protocols are selected based on 
the message size, with each protocol having its own synchronization semantics, which strongly impact performance. SMPI supports different performance modes through a generalization of the LogGPS model. Another difficult issue is to model network topologies and contention. SMPI relies on SimGrid's communication models where each ongoing communication is represented as a whole (as opposed to single packets) by a flow. Assuming steady-state, contention between active communications can then be modeled as a bandwidth sharing problem that accounts for non-trivial phenomena (e.g., crosstraffic interference [16]). If needed, communications that start or end trigger a re-computation of the bandwidth share. In this fluid model, the time to simulate a message passing through the network is independent of its size, which is advantageous for large-scale applications frequently sending large messages and orders of magnitude faster than packet-level simulation. SimGrid does not model transient phenomena incurred by the network protocol but accounts for network topology and heterogeneity. Special attention to the modeling of collective communication algorithms has also been paid in SMPI, but this is of little significance in this article as HPL ships with its own implementation of collective operations.

SMPI maps every MPI rank of the application onto a lightweight simulation thread. These threads are then run one at a time, i.e., in mutual exclusion. Every time a thread enters an MPI call, SMPI takes control and the time that was spent computing (isolated from the other threads) since the previous MPI call is injected into the simulator as a virtual delay. This time may be scaled up or down depending on the speed of the simulated machine with respect to the simulation machine. Recent results report consistent performance predictions within a few percent for standard benchmarks on small-scale clusters (up to $12 \times 12$ cores [17] and up to $128 \times 1$ cores [2]). In this article, we validate this approach at a much larger scale with HPL, whose emulation comes with at least two challenges:

- The time-complexity of the algorithm is $\Theta\left(N^{3}\right)$ and $\Theta\left(N^{2}\right)$ communications are performed, with $N$ being very large. The execution on the Stampede cluster took roughly two hours on 6006 compute nodes. Using only a single node, a naive emulation of HPL at the scale of the Stampede run would take about 500 days if perfect scaling was reached.

- The tremendous memory consumption and amount of memory accesses need to be drastically reduced.

\section{EMUlating HPL AT LARGE SCALE}

We now present the changes to SimGrid and HPL that were required for a scalable simulation. We provide only a brief presentation of our modifications and refer the reader interested in details to a previous report [18], [19] and a companion repository [20]. For our experiments in this section, we used a single core from nodes of the Nova cluster provided by the Grid'5000 testbed [21] (32 GB of RAM, two 8-core Intel Xeon E5-2620 v4 CPUs, Debian Stretch OS (Linux 4.9)).

\section{A. Speeding Up the Emulation}

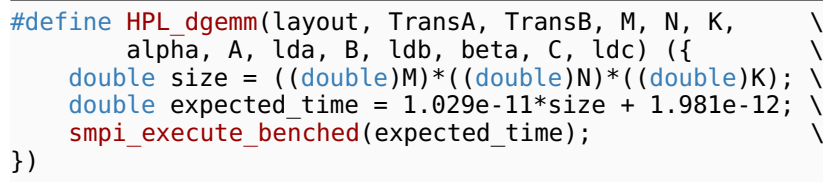

(a) Non-intrusive macro replacement with a very simple computation model.

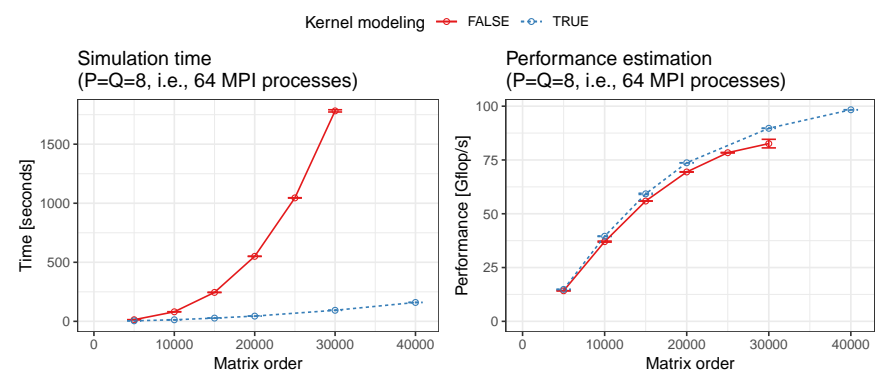

(b) Gain in terms of simulation time.

Figure 3: Replacing the calls to computationally expensive functions by a model allows to emulate HPL at a larger scale.

1) Compute Kernel Modeling: HPL heavily relies on BLAS kernels such as dgemm (for matrix-matrix multiplication) or dtrsm (for solving an $A \cdot x=b$ equation). The analysis of an HPL simulation with 64 processes and a very small matrix of order 30,000 showed that about $96 \%$ of the time is spent in these two kernels. Since the output of these kernels does not influence the control flow, simulation time can be reduced by substituting dgemm and dtrsm function calls with a performance model of the respective kernel. Skipping kernels renders the content of some variables invalid but in simulation, only the behavior of the application and not the correctness of computation results are of concern. Figure 3(a) shows an example of this macro-based mechanism that allows us to keep HPL code modifications to an absolute minimum. The $(1.029 \mathrm{e}-11)$ value represents the inverse of the flop rate for this compute kernel and was obtained through calibration. The estimated time of the kernel is calculated based on the given parameters and passed on to smpi_execute_benched that advances the clock of the executing rank by this estimate. The effect on the simulation time for a small scenario is depicted in Figure 3(b). This modification speeds up the simulation by orders of magnitude. The precision of the simulation will be investigated in more details in the next sections but it can already be observed that this simple kernel model leads to a sound, albeit slightly more optimistic, estimation of the performance.

In addition to the main compute kernels, we identified seven other BLAS functions through profiling as computationally expensive enough to justify a specific handling: dgemv, dswap, daxpy, dscal, dtrsv, dger and idamax. Similarly, a significant amount of time was spent in fifteen functions implemented in HPL: HPL_dlaswp*N, HPL_dlaswp*T, HPL_dlacpy and HPL_dlatcpy. All these functions are called during the LU factorization and hence impact the performance measured by HPL; however, because of the removal of the dgemm and 
dtrsm computations, they all operate on bogus data and hence also produce bogus data. To study their impact on the overall performance, we handled them similarly to dgemm and dtrsm, through performance models and macro substitution, which speeds up the simulation by an additional factor of 3 to 4 on small $(N=30,000)$ and even more on large scenarios.

2) Specific Adjustments: HPL uses pseudo-randomly generated matrices that are setup every time HPL is executed. This initialization, just like the factorization correctness verification at the end of the run, is not considered in the reported performance and can therefore be safely skipped. Note that HPL implements an LU factorization with partial pivoting, which requires a special treatment of the idamax function that returns the index of the first element equaling the maximum absolute value. Although we ignored the cost of this function as well, we set its return value to a random (but controlled) value to make the simulation unbiased (but fully deterministic). We confirmed that this modification was harmless in terms of performance prediction (see [19] for details).

\section{B. Scaling Down Memory Consumption}

The largest two allocated data structures in HPL are the input matrix A (with a size of typically several GB per process) and the panel which contains information about the sub-matrix currently being factorized. This sub-matrix typically occupies a few hundred MB per process. Unfortunately, when emulating an application with SMPI, all MPI processes are run within the same simulation process on a single node and the memory consumption of the simulation can therefore quickly reach several TB of RAM. Yet, as we no longer operate on real data, storing the whole input matrix $A$ is needless. However, since only a minimal portion of the code was modified, some functions may still read or write some parts of the matrix. It is thus not possible to simply remove the memory allocations of large data structures. SMPI provides the SMPI_SHARED_MALLOC (SMPI SHARED FREE) macro to replace calls to malloc (free). They indicate that some data structures can safely be shared between processes and that the data they contain is not critical for the execution (e.g., an input matrix) and that it may even be overwritten. SMPI_SHARED_MALLOC works as follows (see Figure 4): a single block of physical memory (of default size $1 \mathrm{MB})$ for the whole execution is allocated and shared by all MPI processes. A range of virtual addresses corresponding to a specified size is reserved and cyclically mapped onto the previously obtained physical address. This mechanism allows most applications to obtain a nearly constant memory footprint, regardless of the size of the actual allocations.

Although using the default SHARED_MALLOC mechanism works flawlessly for A, a more careful strategy needs to be used for the panel, which is an intricate data structure with both ints (accounting for matrix indices, error codes, MPI tags, and pivoting information) and doubles (corresponding to a copy of a sub-matrix of A). To optimize data transfers, HPL flattens this structure into a single allocation of doubles (see Figure 5(a)). Using a fully shared memory allocation for the panel therefore leads to index corruption that results in classic invalid memory

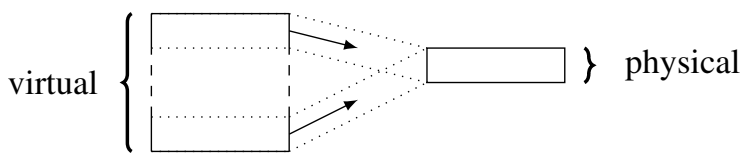

Figure 4: SMPI shared malloc mechanism: large area of virtual memory are mapped onto the same physical pages.

\begin{tabular}{|c|c|c|}
$\overbrace{\text { matrix parts }}^{\text {can be shared }}$ & indices & matrix parts \\
\hline
\end{tabular}

(a) Structure of the panel in HPL. initial buffer

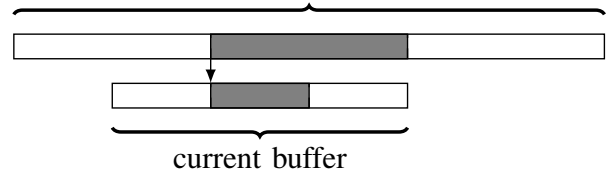

(b) Reusing panel allocation from an iteration to another.

Figure 5: Panel structure and allocation strategy.

accesses. Since ints and doubles are stored in non-contiguous parts of this flat allocation, it is therefore essential to have a mechanism that preserves the process-specific content. We have thus introduced the SMPI_PARTIAL_SHARED MALLOC macro that allows us to specify which ranges of the allocation should be preserved (i.e., are private to each process) and which ones may be corrupted (i.e., are shared between processes). For a matrix of order 40,000 and 64 MPI processes, memory consumption decreases with this approach from about $13.5 \mathrm{~GB}$ to less than $40 \mathrm{MB}$.

Another HPL specific optimization is related to the systematic allocation and deallocation of panels in each iteration, with the size of the panel strictly decreasing from iteration to iteration. As we explained above, the partial sharing of panels requires many calls to mmap and introduces an overhead that makes these repeated allocations / frees a bottleneck. Since the very first allocation can fit all subsequent panels, we modified this allocation mechanism so that SMPI can reuse panels as much as possible from an iteration to an other (see Figure 5(b)). Even for a very small matrix of order 40,000 and 64 MPI processes, the simulation time decreases from $20.5 \mathrm{sec}$ to $16.5 \mathrm{sec}$. The number of page faults decreased from 2 million to 0.2 million, confirming the devastating effect these allocations/deallocations would have at scale.

The last three optimizations we describe are not specific to HPL. We leveraged the information on which memory area is private, shared or partially shared to improve the overall performance. By making SMPI internally aware of the memory's visibility, it can now avoid calling memcopy when large messages containing shared segments are sent from one MPI rank to another. For fully private or partially shared segments, SMPI identifies and copies only those parts that are process-dependent (private) into the corresponding buffers on the receiver side. HPL simulation times and memory consumption were considerably improved in our experiments because the panel is the most frequently transferred data 


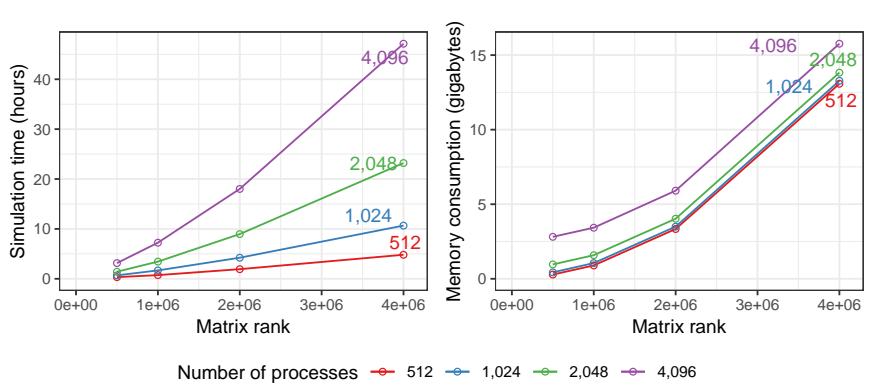

Figure 6: Time complexity and memory consumption are linear in the number of processes but remain mildly quadratic with matrix rank.

structure but only a small part of it is actually private.

As explained above, SMPI maps MPI processes to threads of a single process, effectively folding them into the same address space. Consequently, global variables in the MPI application are shared between threads unless these variables are privatized and the simulated MPI ranks thus isolated from each other. Several technical solutions are possible to handle this issue [2]. The default strategy in SMPI consists in making a copy of the data segment (containing all global variables) per MPI rank at startup and, when context switching to another rank, to remap the data segment via mmap to the private copy of that rank. SMPI also implements another mechanism relying on the dlopen function that allows to load several times the data segment in memory and to avoid costly calls to mmap (and subsequent cache flush) when context switching. For a matrix of order 80,000 and 32 MPI processes, the number of minor page faults drops from 4,412,047 (with mmap) to 6880 (with dlopen), which results in a reduction of system time from $10.64 \mathrm{sec}$ (out of $51.47 \mathrm{sec}$ ) to $2.12 \mathrm{sec}$.

Finally, for larger matrix orders (i.e., $N$ larger than a few hundred thousands), the performance of the simulation quickly deteriorates as the memory consumption rises rapidly. Indeed, folding the memory reduces the physical memory usage. The virtual memory, on the other hand, is still allocated for every process since the allocation calls are still executed. Without a reduction of allocated virtual addresses, the page table rapidly becomes too large for a single node. Thankfully, the x86-64 architecture supports several page sizes, such as the huge pages in Linux. Typically, these pages are around $2 \mathrm{MiB}$ (instead of $4 \mathrm{KiB}$ ), which reduces drastically the page table size. For example, for a matrix of order $N=4,000,000$, it shrinks from $250 \mathrm{~GB}$ to $0.488 \mathrm{~GB}$.

\section{Scalability Evaluation}

The main goal of the previous optimizations is to reduce the complexity from $\Theta\left(N^{3}\right)+\Theta\left(N^{2} \cdot P \cdot Q\right)$ to something more reasonable. The $\Theta\left(N^{3}\right)$ was removed by skipping most computations. Ideally, since there are $N / N B$ iterations (steps), the complexity of simulating one step should be decreased to something independent of $N$. SimGrid's fluid models, used to simulate communications, do not depend on $N$. Therefore, the time to simulate a step of HPL should mostly depend on
$P$ and $Q$. Yet, some memory operations on the panel that are related to pivoting are intertwined in HPL with collective communications, meaning that it is impossible to get rid of the $\mathcal{O}(N)$ complexity without modifying HPL more profoundly.

To evaluate the efficiency of our proposal, we conduct a first evaluation on a non-existing but Stampede resembling platform comprising 4,096 nodes interconnected through a fattree topology. We run simulations with $512,1024,2048$ or 4096 MPI ranks and with matrices of orders $5 \times 10^{5}, 1 \times 10^{6}$, $2 \times 10^{6}$ or $4 \times 10^{6}$. All other HPL parameters are similar to the ones of the original Stampede scenario. The impact of the matrix order on total makespan and memory is illustrated in Figure 6. With all previously described optimizations enabled, the longest simulation took close to 47 hours and consumed $16 \mathrm{~GB}$ of memory whereas the shortest one took 20 minutes and $282 \mathrm{MB}$ of memory. These optimizations also enabled us to simulated the configuration used for the Stampede cluster in 2013 for the TOP500 ranking in less than 62 hours and using $19 \mathrm{~GB}$ on a single node of a commodity cluster (see Table I).

\section{Modeling HPL Kernels And COMmunications}

As explained in Section V, HPL spends most of its computation time in a dozen specific functions for which a performance model has to be designed. Most compute kernels have several parameters from which a very simple model can generally easily be identified (e.g., proportional to the product of the parameters) but refinements including the individual contribution of each parameter as well as the spatial and temporal variability of the operation are also possible. Likewise, communications between two nodes are mostly linear in message size but the actual performance can wildly vary depending on the range of the message size as MPI switches from one protocol to another whenever needed (see Figure 7). In this section we first introduce some notations to describe the complexity of the models we have investigated. We then briefly compare the prediction of these models with individual measurements of both computations and communications to illustrate the importance of the model complexity. We finally show in Section VII that using simpler modeling options leads to a serious inaccuracy of the simulation.

\section{A. Modeling Notations}

We denote as $T$ the duration of an operation with parameters $M, N, K$ (in the case of the dgemm operation, these parameters describe the geometry of the input matrices). We first consider the three following modeling options:

- Modeling option $\mathcal{M}-0$ : For simple and stable compute kernels, the duration can be modeled as a constant duration independent of the input parameters, i.e., $T \sim \alpha$, where $\alpha$ is estimated through the sample average of the duration of the operation (or simply 0 if the kernel is negligible) .

- Modeling option $\mathcal{M}-1$ : A simple combination of the parameters (e.g., $S=M . N . K$ ) may be the primary factor driving the performance of the operation. Then $T \sim \alpha . S(+\beta)$ and $\alpha$ and $\beta$ can be estimated through a classical least-square linear regression. 
- Modeling option $\mathcal{M}-2$ : When the behavior of the operation is complex or requires a faithful modeling over the full range of input parameters, a full polynomial model is required, i.e., $T \sim \alpha . M . N . K+\beta . M . N+\gamma . N . P+\ldots$ Again, the $\alpha, \beta, \gamma, \ldots$ can be estimated through a classical least-square linear regression.

There are two situations where more elaborate variations need to be considered:

- Whenever the platform is slightly heterogeneous (spatial variability), the previous models should be built for each host individually. This modeling option is denoted $\mathcal{M}_{H}$.

- The behavior of the operation may be mostly linear but only for specific parameter ranges. This is for example the case for networking operations or for computing nodes on Stampede where Intel's Math Kernel Library (MKL) uses the Xeon Phi accelerator only when the input is large enough to compensate for the data transfer. In such situations, the models considered will be piece-wise linear,

$$
\text { e.g., } T \sim \begin{cases}\text { if } M<\theta_{1} & \alpha_{1} \cdot M+\beta_{1} \\ \text { else if } M<\theta_{2} & \alpha_{2} \cdot M+\beta_{2}, \\ \ldots & \end{cases}
$$

where the $\theta, \alpha, \beta$ should all be estimated. This kind of model is denoted $\mathcal{M}^{\prime}$.

All previous models can be fit with relatively simple linear regressions or maximum likelihood learning methods. However, an important hypothesis underlying all these methods is the homoscedasticity, i.e., that the variability is independent on the parameters.

The residual (temporal) variability may be an important phenomenon to account for, as "system noise" is known to be detrimental to the overall performance of parallel applications like HPL. We thus consider different modeling options for this temporal variability:

- Noise option $\mathcal{N}-0$ (no noise): This is the simplest option. It consists in injecting the value predicted by the model

- Noise option $\mathcal{N}-1$ (homoscedastic): The simplest probability family to model variability is the normal distribution, hence $T \sim \mathcal{M}(M, N, K)+N\left(0, \sigma^{2}\right)$, where $\sigma^{2}$ is the sample variance of the model residuals.

- Noise option $\mathcal{N}-2$ (heteroscedastic): The conditional variance of the residuals (i.e., $\sigma^{2}$ given $M, N, K$ ) is modeled by a polynomial function of the input parameters.

Finally, even the sophisticated normal distribution from $\mathcal{N}-2$ may be too simple to describe the noise observed on real platforms where it may be common for a same parameter set to have a few operations being one order of magnitude slower than all the other ones. In this case, a reasonable option consists of modeling noise with a mixture of normal distributions whose parameters $\pi_{1}, \ldots, \pi_{k}$ should be estimated. We denote this kind of model as $\mathcal{N}^{\prime}$. Likewise, the per-host estimations are denoted by $\mathcal{N}_{H}$.

Linear regression is a standard tool in $\mathrm{R}$ [22] or in python/statsmodels [23] and models $\mathcal{M}_{H}-0,1,2$ are thus easy to fit assuming $\mathcal{N}-1$. Model $\mathcal{M}^{\prime}-0$ (piece-wise constant) assuming

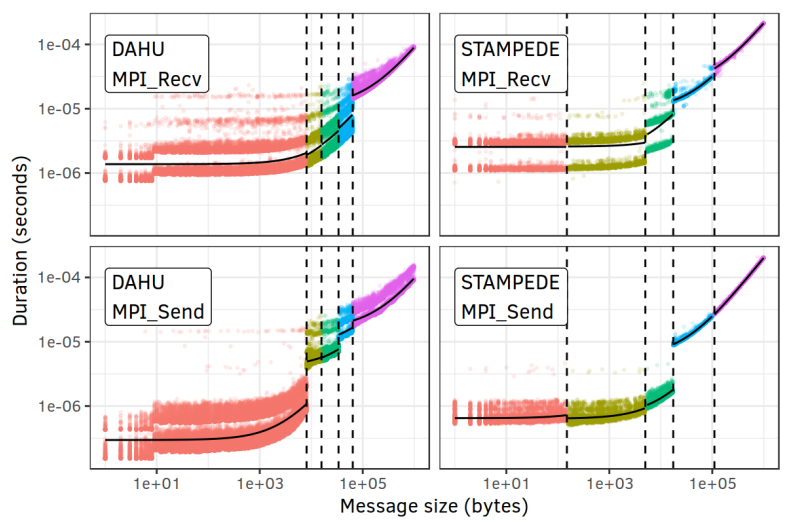

Figure 7: Illustrating piecewise linearity and temporal variability of high-speed communications on two systems.

$\mathcal{N}-1$ can easily be fit using the cubist [24] library. We could not find any implementation allowing to fit $\mathcal{M}^{\prime}-1$ (piece-wise linear, possibly discontinuous) assuming $\mathcal{N}-1$ so we implemented a method based on model trees [25, Chapter 9] [26] in a python library (pytree). When the noise is heteroscedastic $(\mathcal{N}-2)$, it is sometimes possible to fall back to the previous methods if a careful sampling method is employed (by sampling more on highly variable areas and fitting the average). Finally, for even more complex noise modeling options $\left(\mathcal{N}^{\prime}-\right)$, some custom Expectation Maximization algorithms are available (e.g., flexmix [27] for $\left.\mathcal{M}-1 \mathcal{N}^{\prime}-1\right)$.

\section{B. Modeling MPI communications}

Prior to this work, the standard way of accounting for protocol changes in SMPI was to estimate breakpoints visually and to conduct a linear regression for each range. The expected duration was then used directly in the simulation with no particular effort with respect to the temporal variability $\left(\mathcal{M}^{\prime}-1\right.$ $\mathcal{N}-0$ ). Yet, as illustrated in Figure 7, the variability of high speed networks is quite particular. We therefore diligently estimated all the parameters of $\mathcal{M}^{\prime}-1 \mathcal{N}^{\prime}-1$, where each message size range is automatically estimated with pytree, as well as the 2 to 4 modes of the Gaussian mixture for each range. Such temporal variability could explain some (overall bad) performance since they generally get amplified by broadcast and pipelined communication patterns.

\section{Modeling dgemm}

HPL spends the most time in the dgemm kernel. We therefore evaluated the previous modeling alternatives: $\mathcal{M}-\{1,2\}$ $\mathcal{N}-\{0,1,2\}$ and $\mathcal{M}_{H}-\{1,2\} \mathcal{N}_{H}-\{0,1,2\}$. The $\mathcal{M}^{\prime}$ and $\mathcal{N}^{\prime}$ families were not investigated as nothing in our observations called for such complexity on classical multi-core machines. Figure 8 illustrates various models and their respective quality for the dgemm function. In these figures, the performance of dgemm is evaluated by calling dgemm with randomized sizes over all the cores of each node (to reproduce experimental conditions similar to the one of HPL). The first observation (Figure $8(\mathrm{a})$ ) is that a few nodes exhibit quite a different behavior (each color and each regression line under model 


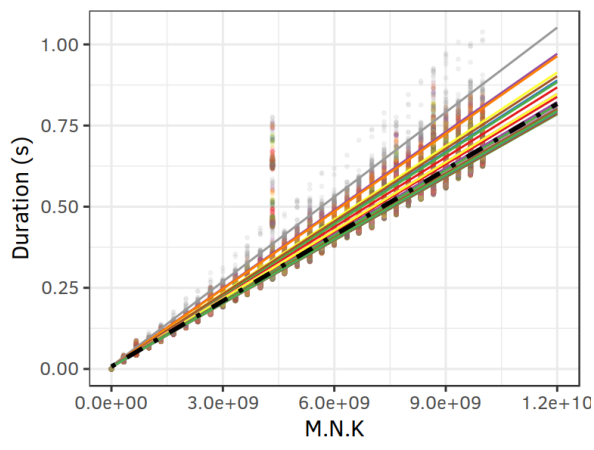

(a) dgemm heterogeneity

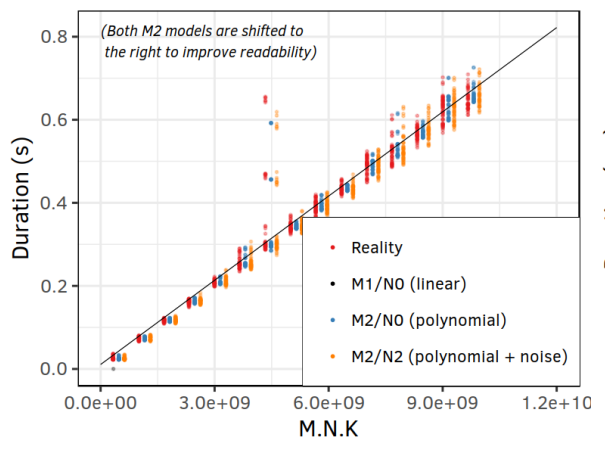

(b) dgemm model

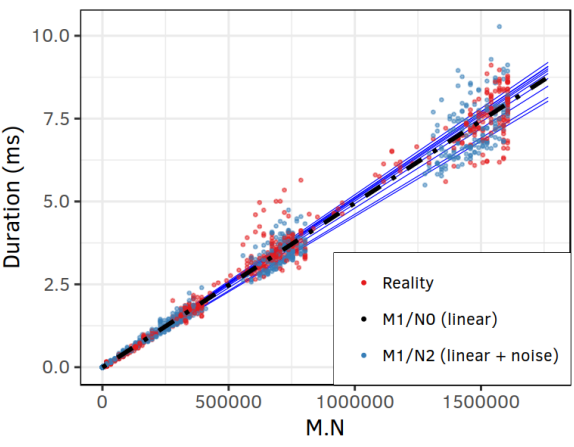

(c) HPL dlatcpy model

Figure 8: Illustrating the realism of modeling for BLAS and HPL functions.

$\mathcal{M}_{H}-1$ corresponds to a different cpu, whereas the black dotted line corresponds to model $\mathcal{M}-1$ over all the nodes). These nodes will systematically be slightly slower than other nodes and accounting for this spatial heterogeneity is likely to be rather important for HPL. Second, we took care of covering a wide variety of combinations for $M, N$, and $K$ and it can be observed that M.N.K is not sufficient to describe correctly the performance of dgemm. Indeed, for M.N.K $\approx 4.5 \times 10^{9}$ some duration are systematically higher regardless of the node. This happens for some particular (e.g., tall and skinny) matrix geometries, which strongly suggests using the full polynomial model. Figure 8 (b) depicts the performance (red dots) of a given node as well as the prediction using a simple linear model $\left(\mathcal{M}_{H}-1\right.$, black line $)$, a full polynomial model $\left(\mathcal{M}_{H}-2\right.$, blue dots) and a full polynomial model with heteroscedastic noise $\left(\mathcal{M}_{H}-2 \mathcal{N}_{H}-2\right.$, orange dots). A close inspection reveals that all experimental variability is actually very well explained by both the polynomial model (better fit for particular parameter combinations) and some temporal variability.

\section{Modeling Other BLAS and HPL Kernels}

Four other BLAS kernels and a few other very small HPL compute kernels (often related to memory management) are deeply intertwined with collective operations to allow HPL to be as efficient as possible. Although the total duration of these kernels is extremely small compared to the total execution time, they may perturb collective communication by introducing late sends and receives. The behavior of one of these kernels is illustrated in Figure 8(c). This kind of data can only be obtained by running HPL for a small input matrix over each node individually. Again, for all these kernels a single parameter combination explains most of the performance and there is some variability from one node to another (one blue regression line per CPU) but it remains quite limited (black dotted line for the platform as a whole), especially since these kernels are very short and infrequently called compared to dgemm. Finally, since variability significantly increases with the value of the input parameters, a $\mathcal{N}-2$ model is clearly required. The blue dots in Figure 8(c) represent the outcome of a $\mathcal{M}-1 \mathcal{N}-2$ model and are hardly distinguishable from the real behavior. Similar results can be obtained with this category of model for all other kernels.

\section{VAlidation AT SCALE}

\section{A. Experimental Setup}

To evaluate the soundness of our approach, we compare several real executions of HPL with simulations using the previous models. We used the Dahu cluster from the Grid'5000 testbed. It has 32 nodes connected through a single switch by $100 \mathrm{Gbits}^{-1}$ Omnipath links. Each node has two Intel Xeon Gold $6130 \mathrm{CPU}$ with 16 cores per CPU and we disabled hyperthreading. We used HPL version 2.2 compiled with GCC version 6.3.0. We also used the libraries OpenMPI version 2.0.2 and OpenBLAS version 0.3.1. HPL executions were done using a block size of 128 and a matrix of varying size (from 50,000 to 500,000 ). We used one single-threaded MPI rank per core and a look-ahead depth of 1 . Finally, we used the increasing-2-ring broadcast with the Crout panel factorization algorithms.

Although this machine is much smaller than top supercomputers, faithfully simulating an HPL execution with such settings is already quite challenging.

- We used one rank per core to obtain a higher number (1024) of MPI process. This is more difficult than simulating one rank per node, as (1) this increases the amount of data transferred through MPI and (2) the performance is then subject to memory interference and network heterogeneity (we used a different model for local and remote communications).

- We used a smaller block size than commonly used, which leads to a higher number of iterations and hence more complex communication patterns.

- We used relatively small input matrices, which reduces the makespan and makes good predictions harder to obtain.

\section{B. Comparing Simulations with Real Executions}

Our first evaluation consists in comparing the traces of the simulations with reality. We instrumented HPL to collect the start and end timestamps of each kernel and MPI call. We limited the execution to 256 ranks and the first five iterations. A first qualitative validation can be done by visually comparing the Gantt charts of the simulations with reality (see Figure 9). Calls to dgemm are depicted in yellow, MPI_Send in red, MPI_Recv in blue. Although the structure is similar in all charts, the shape and the duration of the communication phases can be overly optimistic in simulations compared to reality. 

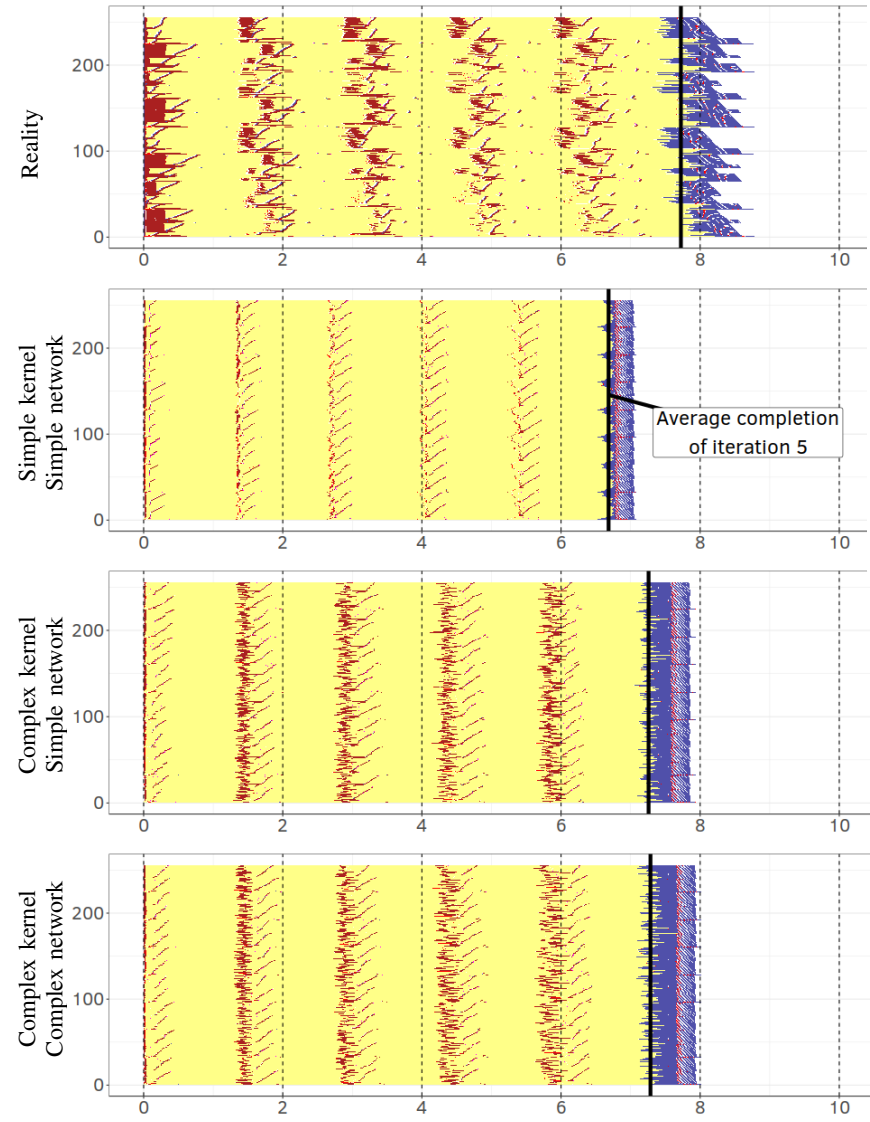

Figure 9: Gantt charts of HPL first iterations in simulation

The charts show that, at this scale, using a deterministic or a stochastic model for the network has no noticeable impact on HPL simulation. However, having a more complex model for the kernels leads to much more realistic traces. The variability in the computation durations leads to an increase of the time spent in communications and an overall slightly longer execution. In HPL, computation variability directly translates to late senders/receivers that destroy the efficiency of collective operations.

We now provide a more quantitative comparison using the whole cluster and varying matrix sizes, focusing on the GFlop s $^{-1}$ rate reported by HPL (see Figure 10). The real executions are depicted in black, for each matrix size we performed 8 runs of HPL, to illustrate the temporal variability of the performance. The line (a), on the top, is our first attempt to simulate HPL. The simulation was done with a simple model: $\mathcal{M}-1$ for the kernels and $\mathcal{M}^{\prime}-1$ for the network with no noise $(\mathcal{N}-0)$ in both cases. This model overestimates HPL performance by more than $30 \%$. We initially thought that the network model was too optimistic, however, switching to a stochastic multi-modal network model $\left(\mathcal{N}^{\prime}-1\right.$, the line (b)), does not significantly improve the prediction precision.

Figure 8 shows that there is an important heterogeneity in the cluster. For this reason, we started using a $\mathcal{M}_{H}-1 / \mathcal{N}-0$ model for dgemm while keeping the models for the other kernels and the network as before. This increases very significantly

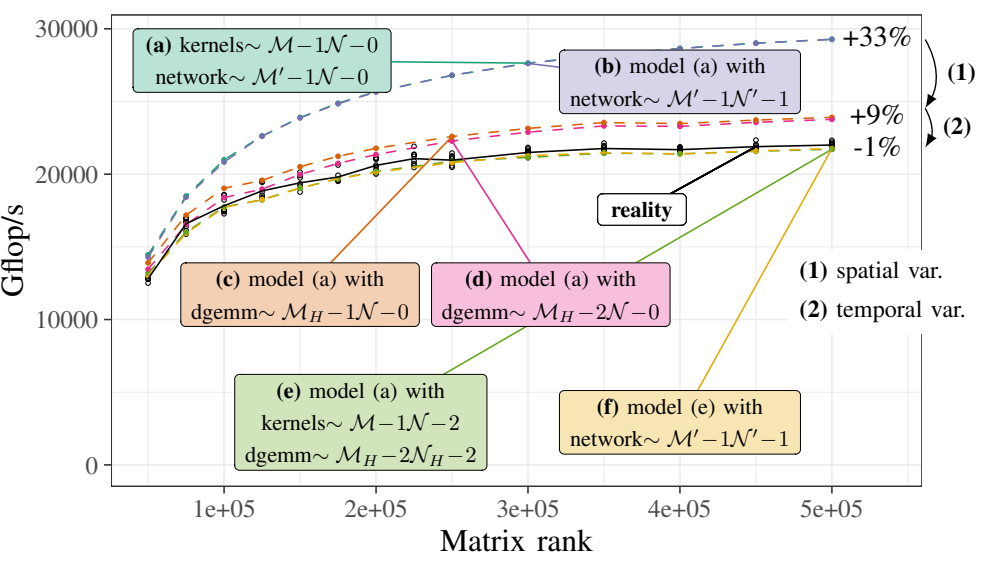

Figure 10: HPL performance: predictions vs. reality

the realism of the simulation as the performance is now overestimated by only $9 \%$ (the line (c)). Using a polynomial model for dgemm instead of a linear model (thus switching from $\mathcal{M}_{H}-1$ to $\mathcal{M}_{H}-2$ ) further improves the performance prediction, in particular for smaller matrices. This new model (the line (d)), is very close to reality at the beginning but becomes equivalent to the previous model for larger matrices. We found that adding the temporal variability noise $(\mathcal{N}-2$ for all kernels, $\mathcal{N}_{H}-2$ for dgemm) is the key ingredient to obtain the last bit of realism. The prediction (the line (e)) is now extremely close to reality as it slightly underestimates the performance by less than $5 \%$ and even as little as $1 \%$ for the larger matrices. Adding back temporal variability to the network model $\left(\mathcal{N}^{\prime}-1\right.$, line (f)) still has no significant effect but this can be explained by the fact that HPL mostly communicates very large amounts of data in bulk. Network temporal variability is however very important aspect to model for applications that are more latency-bound.

As illustrated in Figure 10 (rightmost labels), we would like to stress again that accurate predictions require a careful modeling of both spatial and temporal variability. Overall, although this was not particularly foreseeable and could have been different with an other application, only the dgemm kernel needs to be carefully modeled with a $\mathcal{M}_{H}-2-\mathcal{N}_{H}-2$. In Figure 1, we used a simple $\mathcal{M}-1-\mathcal{N}-0$ for the other BLAS kernels and simply skipped every other kernel. A detailed modeling of all (BLAS and HPL) kernels is possible but a minimal calibration of the dgemm kernel over a representative set of nodes is thus sufficient to consistently predict the performance of HPL on this machine within a few percent of reality.

\section{Comparing With the Stampede Qualification Run}

Each node of the Stampede cluster comprises two 8-core Intel Xeon E5-2680 8C 2.7 GHz CPUs and one 61-core Intel Xeon Phi SE10P (KNC) $1.1 \mathrm{GHz}$ accelerator that is roughly three times more powerful than the two CPUs. The HPL output submitted to the TOP500 (Table II) does not indicate how the KNC was used. However, because of the values assigned to $P$ and $Q$, we are certain that there were only one MPI rank 
per node. For this reason, it is likely that the KNC was used as an accelerator, which is effortless with Intel's Math Kernel Library (MKL) as it supports automatic offloading for selected BLAS functions. While we cannot know precisely which MKL version was used in 2013, we measured the default version (version 11.1.1) that was used on Stampede in the beginning of 2017 and built precise $\mathcal{M}^{\prime}-1 \mathcal{N}-1$ models of dgemm and dtrsm. Likewise, using measurements of the performance of MPI obtained in 2017 , we built a precise $\mathcal{M}^{\prime}-1 \mathcal{N}-0$ model of the fat-tree interconnect. All HPL input parameters were set to exactly the values used in the TOP500 qualification run and were coherent with the submitted output.

Surprisingly, despite our efforts, our predictions forecast a significantly lower performance $(\approx 20-30 \%)$ than the one reported for the top500, even when considering an ideal network (no contention, no latency, no noise) and perfectly stable and homogeneous nodes where Xeon Phi would operate at peak performance. Unfortunately, when tracking down the cause of this discrepancy, we eventually realized that despite the reported output which mentioned HPLinpack 2.1 from 0ctober 26, 2012 by Petitet et al., the TOP500 performance had not been obtained with the open-source version of HPL but with a specifically optimized binary version from Intel employing a custom broadcast algorithm relying on nonblocking sends, and which was a priori specifically modified for Stampede. We refer to a previous work [28] for a detailed discussion on this investigation, which explains why this failure to predict similar performance cannot be considered as an invalidation of our approach. Validation experiments therefore cannot rely on the lacunar information provided by the TOP500 and cannot make the economy of a large-scale controlled (software environment, source code) run.

\section{CONCLUSIONS}

Studying and tuning HPC applications at scale can be very time- and resource-consuming. We believe that being capable of precisely predicting an application's performance on a given platform is useful for application developers and users (e.g., to evaluate the scalability of the application on a given machine) and will become invaluable in the future as it can for example aid compute centers with the decision of whether the envisioned technology of a new machine works best for a given application or if an upgrade of the current machine should be considered. Simulation is often an effective approach in this context and SimGrid/SMPI has previously been successfully validated in several small-scale studies with simple HPC benchmarks [2], [17]. In this article, we proposed and evaluated extensions to SMPI that allowed us to emulate HPL at the scale of a supercomputer. Our application of choice, HPL, is particularly challenging both in terms of tuning and simulation as it implements its own set of non-blocking collective operations (from which the users has to choose) that rely on MPI_Iprobe in order to overlap with computations.

More specifically, we explain how to simulate on a single commodity node scenarios at the scale of the TOP500 qualification runs. Although simulation is generally longer than a real execution, it is significantly less resource consuming. This emulation employs several non-trivial operating-system level optimizations (memory mapping, dynamic library loading, huge pages) that have since been integrated into the last version of SimGrid/SMPI. Unlike the common skeleton approach [14], we do not rely on a simplified version of HPL but only apply minor modifications (HPL comprises $16 \mathrm{~K}$ lines of ANSI C over 149 files, our modifications only changed 14 files with 286 line insertions and 18 deletions) to the original source code to fully capture its whole complexity.

We also explain how different levels of detail of modeling of the performance of compute kernels and of the MPI library influence the quality of the prediction. In particular, we show that a careful modeling (including both spatial and temporal variability) of the main compute kernel (dgemm, which is rather inexpensive from a benchmarking perspective) allows to predict performance within a few percents of real experiments with 1,024 ranks on a controlled cluster of Grid'5000. Validation experiments involving larger platforms are underway but, as briefly explained with the Stampede study, cannot be done simply from the information reported in the TOP500 and require access to a well-controlled supercomputer.

As we explained, a careful modeling of networking and computing resources is crucial but requires a diligent combination of statistical libraries. We intend to automate our benchmarking and modeling approach as much as possible using a generic and unified Bayesian estimation framework like STAN [29]. The MCMC sampling technique used in STAN would be particularly helpful to inject uncertainty (in particular the platform heterogeneity which should be estimated from only a few sample nodes) in the simulation. This integration effort is underway. As another future work, building on the effort of SimGrid developers on supporting the emulation of a wide variety of applications with SMPI [30], we also intend to conduct similar studies with other HPC benchmarks (e.g., HPCG [31] or HPGMG [32]), real applications (e.g., BigDFT [33]) and larger infrastructures.

\section{Acknowledgments}

Experiments presented in this paper were carried out using the Grid' 5000 testbed, supported by a scientific interest group hosted by Inria and including CNRS, RENATER and several Universities as well as other organizations (see https://www. grid5000.fr).

We warmly thank our TACC colleagues for their support in this study and providing us with as much information as they could. We thank our Cray colleagues for providing us with information about the TOP500 run of Theta, a resource of the Argonne Leadership Computing Facility, which is a DOE Office of Science User Facility supported under Contract DEAC02-06CH11357.

Last, we warmly thank the SimGrid developers for their help in integrating our contributions as well as for their early feedback on this work before submission. 


\section{REFERENCES}

[1] H. Casanova, A. Giersch, A. Legrand, M. Quinson, and F. Suter, "Versatile, Scalable, and Accurate Simulation of Distributed Applications and Platforms," Journal of Parallel and Distributed Computing, vol. 74, no. 10, pp. 2899-2917, 2014.

[2] A. Degomme, A. Legrand, G. Markomanolis, M. Quinson, M. S. Stillwell, and F. Suter, "Simulating MPI applications: the SMPI approach," IEEE Transactions on Parallel and Distributed Systems, vol. 28, no. 8, pp. 2387-2400, Feb. 2017.

[3] H. W. Meuer, E. Strohmaier, J. Dongarra, and H. D. Simon, The TOP500: History, Trends, and Future Directions in High Performance Computing, 1st ed. Chapman \& Hall/CRC, 2014.

[4] A. Petitet, C. Whaley, J. Dongarra, A. Cleary, and P. Luszczek, "HPL a portable implementation of the High-Performance Linpack benchmark for distributed-memory computers," http://www.netlib.org/benchmark/ hpl, February 2016, version 2.2.

[5] P. Luszczek and J. Dongarra, "Reducing the time to tune parallel dense linear algebra routines with partial execution and performance modeling," in Parallel Processing and Applied Mathematics. Springer Berlin Heidelberg, 2012, pp. 730-739. [Online]. Available: https: //doi.org/10.1007\%2F978-3-642-31464-3_74

[6] G. Zheng, G. Kakulapati, and L. Kale, "BigSim: A Parallel Simulator for Performance Prediction of Extremely Large Parallel Machines," in Proc. of the 18th IPDPS, 2004.

[7] R. M. Badia, J. Labarta, J. Giménez, and F. Escalé, "Dimemas: Predicting MPI Applications Behaviour in Grid Environments," in Proc. of the Workshop on Grid Applications and Programming Tools, Jun. 2003.

[8] M. Mubarak, C. D. Carothers, R. B. Ross, and P. H. Carns, "Enabling parallel simulation of large-scale HPC network systems," IEEE Transactions on Parallel and Distributed Systems, 2016.

[9] H. Casanova, F. Desprez, G. S. Markomanolis, and F. Suter, "Simulation of MPI applications with time-independent traces," Concurrency and Computation: Practice and Experience, vol. 27, no. 5, p. 24, Apr. 2015. [Online]. Available: https://hal.inria.fr/hal-01232776

[10] X. Wu and F. Mueller, "ScalaExtrap: Trace-based communication extrapolation for SPMD programs," in Proc. of the 16th ACM Symp. on Principles and Practice of Parallel Programming, 2011, pp. 113-122.

[11] L. Carrington, M. Laurenzano, and A. Tiwari, "Inferring large-scale computation behavior via trace extrapolation," in Proc. of the Workshop on Large-Scale Parallel Processing, 2013.

[12] C. L. Janssen, H. Adalsteinsson, S. Cranford, J. P. Kenny, A. Pinar, D. A. Evensky, and J. Mayo, "A simulator for large-scale parallel architectures," International Journal of Parallel and Distributed Systems, vol. 1, no. 2, pp. 57-73, 2010, http://dx.doi.org/10.4018/jdst.2010040104.

[13] C. Engelmann, "Scaling To A Million Cores And Beyond: Using LightWeight Simulation to Understand The Challenges Ahead On The Road To Exascale," FGCS, vol. 30, pp. 59-65, Jan. 2014.

[14] R. F. Bird, S. A. Wright, D. A. Beckingsale, and S. A. Jarvis, "Performance modelling of magnetohydrodynamics codes," in Computer Performance Engineering, M. Tribastone and S. Gilmore, Eds. Berlin, Heidelberg: Springer Berlin Heidelberg, 2013, pp. 197-209.

[15] T. Grass, C. Allande, A. Armejach, A. Rico, E. Ayguadé, J. Labarta, M. Valero, M. Casas, and M. Moreto, "Musa: A multi-level simulation approach for next-generation hpc machines," in Proceedings of the International Conference for High Performance Computing, Networking, Storage and Analysis, ser. SC '16. Piscataway, NJ, USA: IEEE Press, 2016, pp. 45:1-45:12. [Online]. Available: http://dl.acm.org/citation.cfm?id=3014904.3014965

[16] P. Velho, L. Schnorr, H. Casanova, and A. Legrand, "On the Validity of Flow-level TCP Network Models for Grid and Cloud Simulations," ACM Transactions on Modeling and Computer Simulation, vol. 23, no. 4, p. 23, Oct. 2013.

[17] F. C. Heinrich, T. Cornebize, A. Degomme, A. Legrand, A. CarpenAmarie, S. Hunold, A.-C. Orgerie, and M. Quinson, "Predicting the Energy Consumption of MPI Applications at Scale Using a Single Node," in Proc. of the 19th IEEE Cluster Conference, 2017. [Online]. Available: https://hal.inria.fr/hal-01523608

[18] T. Cornebize, "Capacity Planning of Supercomputers: Simulating MPI Applications at Scale," Master's thesis, Grenoble INP ; Université Grenoble - Alpes, Jun. 2017. [Online]. Available: https: //hal.inria.fr/hal-01544827

[19] Tom Cornebize, "Laboratory Notebook hosted on GitHub. Capacity Planning of Supercomputers: Simulating MPI Applications at Scale,"
2017. [Online]. Available: https://github.com/Ezibenroc/m2_internship_ journal

[20] T. Cornebize, A. Legrand, and F. C. Heinrich, "Fast and Faithful Performance Prediction of MPI applications: the HPL use case study (experiment artifact)," May 2019. [Online]. Available: https: //doi.org/10.5281/zenodo.3066213

[21] D. Balouek, A. Carpen-Amarie, G. Charrier, F. Desprez, E. Jeannot, E. Jeanvoine, A. Lèbre, D. Margery, N. Niclausse, L. Nussbaum, O. Richard, C. Pérez, F. Quesnel, C. Rohr, and L. Sarzyniec, "Adding virtualization capabilities to the Grid'5000 testbed," in Cloud Computing and Services Science, ser. Communications in Computer and Information Science, I. Ivanov, M. Sinderen, F. Leymann, and T. Shan, Eds. Springer International Publishing, 2013, vol. 367.

[22] $\mathrm{R}$ Core Team, $R:$ A Language and Environment for Statistical Computing, R Foundation for Statistical Computing, Vienna, Austria, 2018. [Online]. Available: https://www.R-project.org/

[23] S. Seabold and J. Perktold, "Statsmodels: Econometric and statistical modeling with python," in 9th Python in Science Conference, 2010.

[24] M. Kuhn and R. Quinlan, Cubist: Rule-And Instance-Based Regression Modeling, 2018, $\mathrm{R}$ package version 0.2.2. [Online]. Available: https://CRAN.R-project.org/package=Cubist

[25] T. Hastie, R. Tibshirani, and J. H. Friedman, The elements of statistical learning: data mining, inference, and prediction, 2nd Edition, ser. Springer series in statistics. Springer, 2009. [Online]. Available: http://www.worldcat.org/oclc/300478243

[26] D. Malerba, F. Esposito, M. Ceci, and A. Appice, "Top-down induction of model trees with regression and splitting nodes," IEEE Transactions on Pattern Analysis and Machine Intelligence, vol. 26, no. 5, pp. 612-625, may 2004. [Online]. Available: https://doi.org/10.1109\%2Ftpami.2004.1273937

[27] B. Grün and F. Leisch, "FlexMix version 2: Finite mixtures with concomitant variables and varying and constant parameters," Journal of Statistical Software, vol. 28, no. 4, pp. 1-35, 2008. [Online]. Available: http://www.jstatsoft.org/v28/i04/

[28] T. Cornebize, F. C. Heinrich, A. Legrand, and J. Vienne, "Emulating High Performance Linpack on a Commodity Server at the Scale of a Supercomputer," Dec. 2017, working paper or preprint. [Online]. Available: https://hal.inria.fr/hal-01654804

[29] B. Carpenter, A. Gelman, M. D. Hoffman, D. Lee, B. Goodrich, M. Betancourt, M. Brubaker, J. Guo, P. Li, and A. Riddell, "Stan: A probabilistic programming language," Journal of Statistical Software, vol. 76, no. 1, 2017. [Online]. Available: https://doi.org/10.18637\% 2Fjss.v076.i01

[30] S. Developers, "Continuous integration of ECP Proxy Apps, CORAL and Trinity-Nersc benchmarks with SimGrid/SMPI," https://github.com/ simgrid/SMPI-proxy-apps/, 2019.

[31] J. J. Dongarra, M. A. Heroux, and P. Luszczek, "HPCG benchmark : a new metric for ranking high performance computing systems," Technion Israel Institute of Technology, Tech. Rep. UT-EECS-15-736, 2015.

[32] M. F. Adams, J. Brown, J. Shalf, B. Straalen, E. Strohmaier, and S. Williams, "Hpgmg 1.0: A benchmark for ranking high performance computing systems," LBNL, Tech. Rep., 052014.

[33] L. Genovese, A. Neelov, S. Goedecker, T. Deutsch, S. A. Ghasemi, A. Willand, D. Caliste, O. Zilberberg, M. Rayson, A. Bergman, and R. Schneider, "Daubechies wavelets as a basis set for density functional pseudopotential calculations," Journal of Chemical Physics, vol. 129, 2008. 